\title{
Study of EMC Optimization of Automotive Electronic Components using ECAE
}

\author{
Tae-Ho Kim*, Mi-Ro Kim**, and Sang-Yong Jung***
}

\begin{abstract}
As more vehicles become equipped with advanced electronic control systems, more consideration is needed with regards to automotive safety issues related to the effects of electromagnetic waves. Unwanted electromagnetic waves from the antenna, electricity and other electronic devices cause the performance and safety problem of automotive components. In general, Power Integrity and Signal Integrity analysis have been widely used, but these analyses have stayed PCB level. PCB base analysis is different from radiated emission TEST condition so its results are used just for reference. This paper proposes EMC optimization technology using module level 3-dimensional radiation simulation process closed to fundamental test conditions. If module level EMC analysis, which is proposed in this study, is applied to all automotive electronics systems, unexpected EMC noise will be prevented.
\end{abstract}

Keywords: Power Integrity (PI), Signal Integrity (SI), Electromagnetic Compatibility (EMC), Automotive Electronic Component, ECAE (Electronic Computer Aided Engineering)

\section{Introduction}

In recent times, as the hybrid and smart vehicles have been increasingly under development, the increased use of electronic components generate the electromagnetic interferences, so a number of effort to solve the electromagnetic compatibility (EMC) issues has been made at an early stage of development. As the electric modules are diversified with hybrid and electric vehicles, and they are high-frequency driven, so the EMC issues caused by the noise become more and more increasing. In order to restrict noise transfer, isolating by distances are required among noise sources within PCB, harness and receiver. In addition to noise isolation by using arrangement methods, appropriate grounding and shielding techniques for reducing noise are also required so as to reduce noise signals in the high-speed communications.

The studies on shielding effects arising from the noise issues of electronic parts have been continuously made. However, the studies that have been typically made are on the shielding effects by the frequencies in relation to each

\footnotetext{
* CAE Team, Hyundai Mobis, Korea.

(kth1016@mobis.co.kr)

** CAE Team, Hyundai Mobis, Korea. (mrkim@mobis.co.kr)

*** Dept. of Information and Communication Engineering,

Sungkyunkwan University, Korea (syjung@ece.skku.ac.kr)
}

Received 21 April 2014; Accepted 22 May 2014 material property or on the electric field distribution in relation to the housing hole-position. [1]-[5]

As a general, basic simulation to solve this type EMC issue is power Integrity and signal integrity but the techniques are yet limited to the PCB level.

In this paper, EMC characteristics of PCB level to define the noise source of Parking Guide System (PGS) are introduced. Next we describe EMC analysis method considering the effect of the shield-can. Near-field and farfield result also introduced using module level prediction method. Finally, this paper ends with a conclusion and further works. Every prediction method is performed with 3-dimensional commercial SW EMC Studio (EMCos).

\section{Analysis of PCB level EMC}

In this chapter, as a first step for analyzing EMC performance of the PGS system, the location and level of the noise source in PCB is measured using a network analyzer and near-field scanner to analyze the noise sources in the system.

The near-field noises generated at the PCB level should be analyzed along with the factors of surrounding ground and wiring, etc. Further, as the near-field noise analyzed in this manner are used as the noise source of full module or system, thorough understanding has to be followed. 


\subsection{Near-field measurement}

Near-field measurement is a method for scanning the noise on $\mathrm{PCB}$ with electric probe and spectrum analyzer. The probe used for the measurements here is the vertical $\mathrm{H}$ field probe. The frequency and amplitude measured by the EMC Scanner could be seen through the spectrum analyzer.

The measuring frequency band has to be same as simulation step. The measured frequency bandwidth, in this study, has been set as $2 \mathrm{MHz} \sim 1000 \mathrm{MHz}(2 \mathrm{MHz}$ step sweep) and video bandwidth has been set as $100 \mathrm{KHz}$. In this way, the measured values at the spectrum analyzer are used as the source of near-field simulation. In order to obtain the accurate magnetic field intensity, the conversion factor of the magnetic field to consider the state of the real probe has to be added to the voltage data measured at the spectrum.

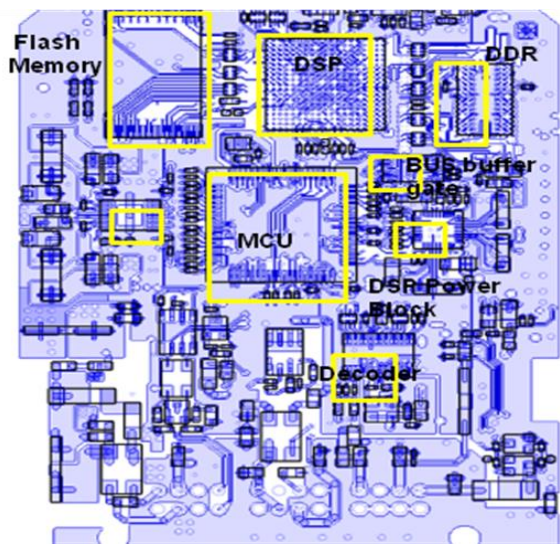

Fig. 1. Distribution of main element at PGS

\subsection{Definition of noise source of PCB}

Fig. 1 shows the schematic design of the PGS board used for this study. Definition of major critical nets of IC and understanding of entire operating system are important for simulation reliability.

In general, the noise source measured by the EMC Scanner could be divided into the internal noise emitted from major ICs, power source noise generated from power side and external noise due to surrounding environment. As for the internal noise sources, there are multiplying frequency of $8 \mathrm{MHz}$ and $27 \mathrm{MHz}$ oscillator elements, high level power source of overall frequency band and operating frequency of main IC. As noted in Fig. 2, the characteristic frequency of the oscillator shows even harmonics and $27 \mathrm{MHz}$ harmonics of the decoder IC.

In principle, measurement process of EMC has to be performed with anechoic chamber to block electromagnetic interference, but we conducted in normal testing labs. From this reason, noise caused by the surrounding environment has been generated regardless of the PCB operating conditions. Consequently as this $900 \mathrm{MHz}$ band source is not related with PCB, the corresponding frequency band has been excluded in our simulation.

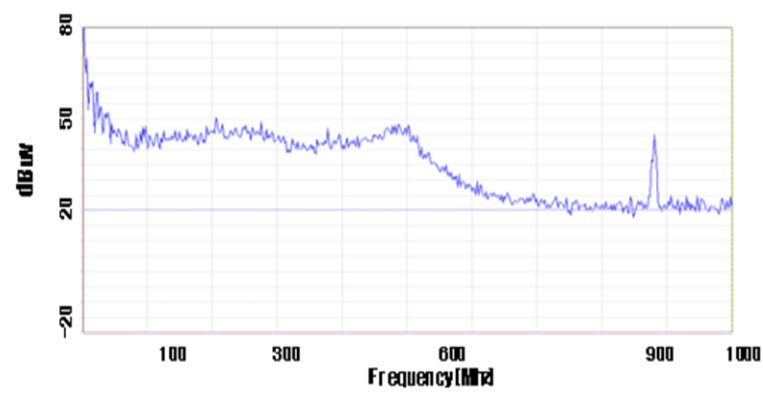

< Decoder IC DATA 27MHz : Harmonic frequency > $<$ Power noise >

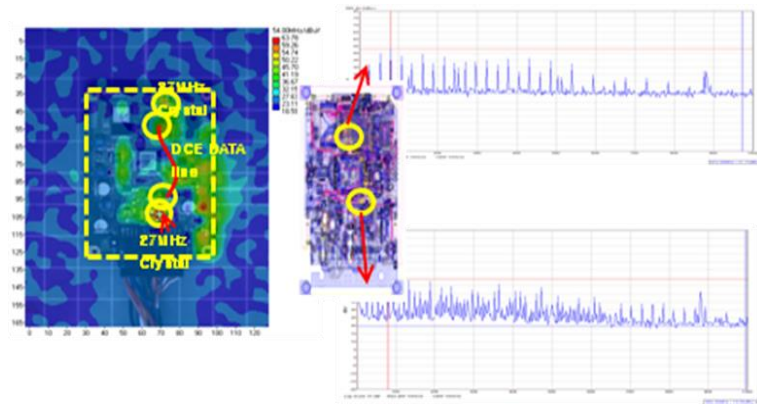

Fig. 2. Noise source of the PGS board

\section{Study of the shield-can effect}

As can be seen in Fig. 3, the metal shield-can is located between housing and PCB. Because of its material property, the effect of shield-can is dominant compared with plastic housing.

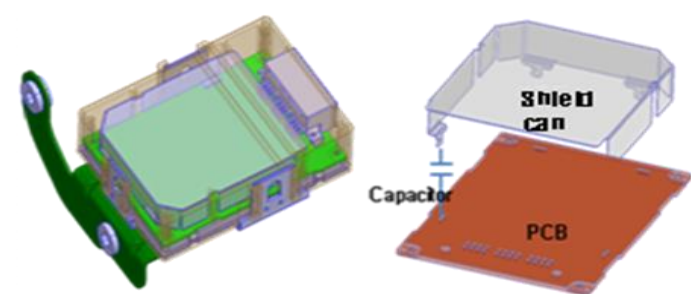

Fig. 3. Structure of the shield-can and PCB of PGS

The role of shield-can is shielding against the noise source generated from PCB. Actually, PCB and shield-can are interconnected with via capacitor, so the capacity value between shield-can and ground plane as well as shape of shield-can could affect the emitted noise level from 
modules. First, in order to predict the ground effect, the simple simulation tests are carried out with shorted condition. The result shows that the resonance impedance occurred at frequency of $294 \mathrm{MHz}, 393 \mathrm{MHz}, 624 \mathrm{MHz}$ and $771 \mathrm{MHz}$.

Proper use of interconnection capacitor between PCB and shield-can could move the resonance frequency of the PGS module to high band and reduce its radiated emission. In this study, to analyze the effect of capacitor value, Z11 simulation has been performed with various cases as shorted, $0.2 p F, 2 p F, 20 p F, 2 n F, 2 u F$. Fig. 4 shows the result of Z11; the ground effect between the shield-can and PCB with various capacitor values.

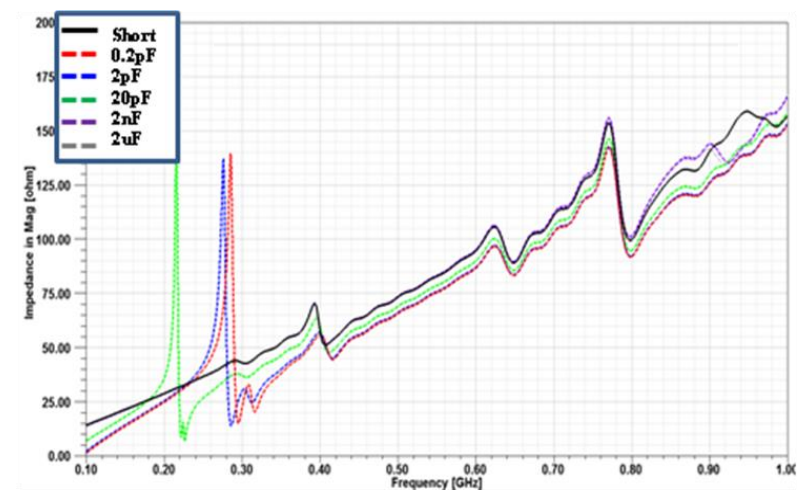

Fig. 4. The grounding effect of capacitor value

From this, in case of the capacitor values below $20 \mathrm{pF}$, we could see impedance level decrease in high frequency band, while resonance occurs near or below $300 \mathrm{MHz}$ band. In case of the capacitor values over $2 \mathrm{uF}$, though overall impedance level is high but no resonance occurs. As a consequence of simulation, the value of capacitor has to be over than $2 \mathrm{uF}$ to stabilize EMC performance of the PGS system with shield-can.

\section{Module level analysis}

In order to analyze the EMC characteristics of PGS module, accurate measuring data of emitting noise from PCB have to be prepared. Then module level simulation could be carried out with taking consideration of the shieldcan and housing conditions into account. Furthermore, if simulation model includes cables, more accurate result will be obtained. Fig. 5 outlines brief explanations on the analysis of the EMC at module level. Fig. 6 is the result of near-field simulation of the PGS module. The first figure indicates analysis model for PGS simulation. Three cases, no shield-can, shorted and connected shield-can with PCB, are checked for analysis. Predicted result of near-field of no shield-can appears that hot spots are prevailed overall region of the PGS board. The shorted case shows hot spots are distributed the side of module, but the level of the middle area, where the shield-can stays, is much reduced. Lastly, when the PCB and chassis ground are connected, most of high level emission is eliminated.

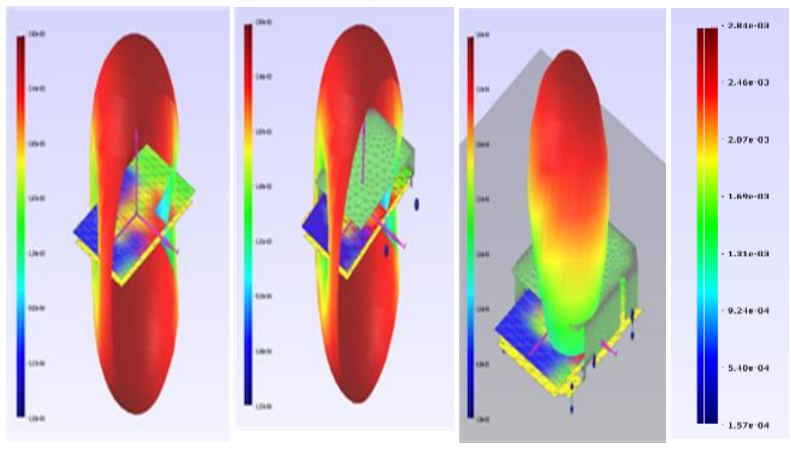

Fig. 5. Overview of EMC analysis method of module level

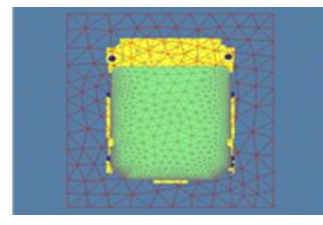

1) mesh model

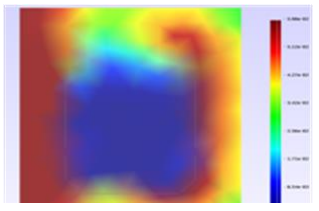

3) shorted shield-can

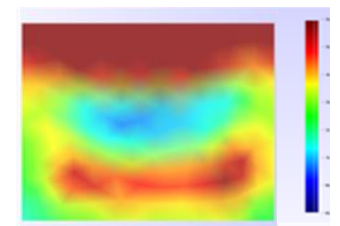

2) without shield-can

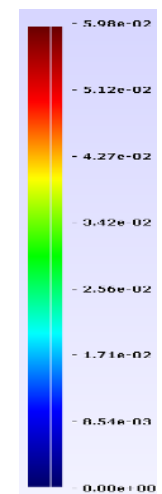

4) with shield-can

Fig. 6. Distribution of near electric filed

Fig. 7 shows the result of the far-field simulation of the PGS module. Compare shorted case with no shield-can, noise emission level of shield-can region decrease slightly. The result of connected case shows that no emissions are radiated to the ground. Furthermore, emissions of upward direction are reduced to a large extent.
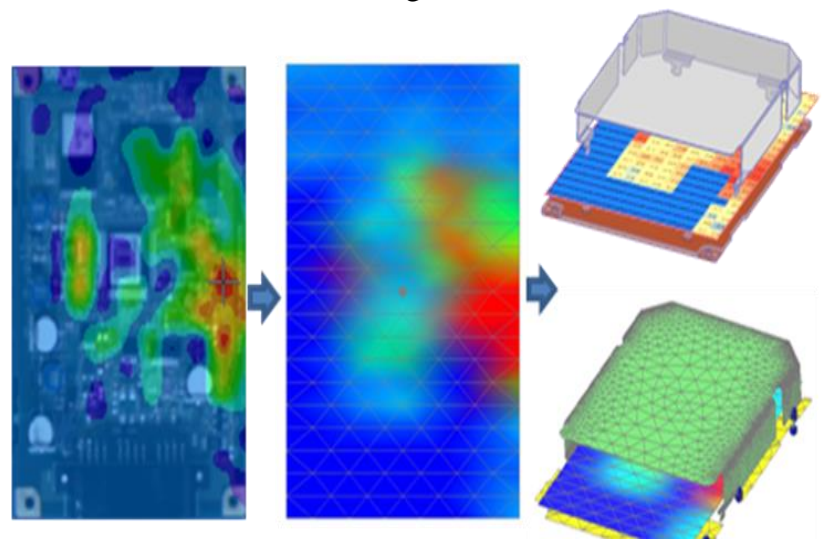

Fig. 7. Distribution of far electric field 
The table 1 shows the maximum value of near-field and far-field. As a result, the case of no shield-can has the highest noise level both near-field and far-field, while the case of connected shows the most stabilized result. In addition, in the PCB Noise frequency which is defined as noise, shield-can effect is less efficient than chassis ground.

In order to obtain more reliable simulation result, most of component at real PCB has to be considered.

Table 1. Maximum radiation value of near / far Field (dBi)

\begin{tabular}{|c|c|c|c|}
\hline & without cover & Shorted cover & With cover \\
\hline $\begin{array}{c}\text { Near Field } \\
\text { Max value }\end{array}$ & $2.712373 \mathrm{e}-1$ & $2.001613 \mathrm{e}-1$ & $5.978851 \mathrm{e}-2$ \\
\hline $\begin{array}{c}\text { Far Field } \\
\text { Max value }\end{array}$ & $2.823190 \mathrm{e}-3$ & $2.741223 \mathrm{e}-3$ & $3.635505 \mathrm{e}-4$ \\
\hline
\end{tabular}

\section{Conclusion}

In general, design of high frequency operating system, such as electrical components, should be carefully handled because shielding effect comes different depending on structure of shield-can and frequency range. In order to make systematic approaches to solve noise issues, analyzing the mechanism of noise inside the car is important. In this paper, to analyze shielding effect and cover resonance, module level simulation has been performed. From that base, module level EMC simulation process is established.

If such EMC performance prediction techniques are expanded to module level at the advanced stage of development, we are sure that much more stabilized system design could be possible. Also, through such advanced prediction analysis, it will contribute not only to shortening the development period, but also to reducing the manufacturing times; thereby it becomes possible that the development costs are reduced.

\section{References}

[1] CHRIS BANYAI, DARYL, DARYL GERKE, "EMI Design Techniques for Microcontrollers in Automotive Applications", Intel, AP-711

[2] H.-H. Chuang, W.-D. Guo , Y.-H. Lin , H.-S. Chen, Y-C Lu, J. Hong , C.-H. Yu , A. Cheng, J. Chou, C.-J. Chang, J. Ku, T.-L. Wu and R.-B. Wu "Signal/power integrity modeling of high-speed memory modules using chip-package-board coanalysis", IEEE Trans. Electronmagn. Compat., vol. 52, no. 2, pp.381 -3912010

[3] "H. W. Ott and C. R. Paul, Eds. (1992). EMC Education Manual, Piscataway: The Education Committee of the IEEE
EMC Society, [Online]. Available:", [online] Available: http://ewh.ieee.org/soc/emcs/pdf/EMCman.pdf

[4] Laleh Golestani-Rad and Jalil Rashed-Mohassel, "The Effects of Apertures' Shape and Configuration on the Shielding Effectiveness of Metallic Enclosures," Proc. of Asia-Pacific Microwave Conference, vol. 5, pp. 3101-3104, Dec. 2005., Virginia, October.

[5] M. P. Robinson, T. M. Benson, C. Christopoulos, J. F. Dawson, M. D. Ganley, A. C. Marvin, S. J. Porter, and D. W. P. Thomas, "Analytical formulation for the shielding effectiveness of enclosures with apertures," IEEE Trans. vol. 40, no. 3, pp. 240-248, Aug. 1998.

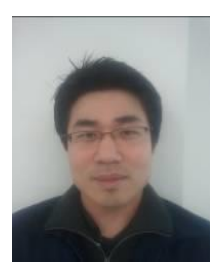

Tae-Ho Kim received M.S degree in electrical engineering from Dongguk university. And $\mathrm{He}$ has working as a Senior Research Engineer of CAE Team in the Hyundai Mobis, Korea. His research interests are EMI/EMC of Automotive Electrical components.

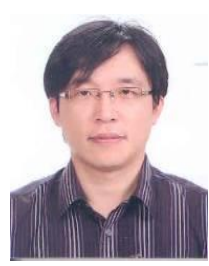

Mi-Ro Kim has working as a Chief Research Engineer of CAE Team in the Hyundai Mobis, Korea. His research interests are EMI/EMC, ESD, Computational Fluid Dynamics(CFD) of Automotive components.

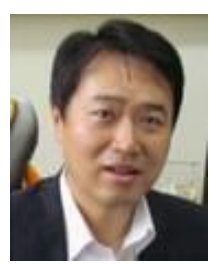

Sang-Yong Jung received B.S., M.S., and Ph.D. degree in electrical engineering from Seoul National University, Seoul, Korea. He was Senior Research Engineer with the R\&D Division, Hyundai Motor Company, Korea. $\mathrm{He}$ is currently an Associate Professor with the College of Information and Communication Engineering, Sungkyunkwan University, Suwon, Korea. His research interests include the numerical analysis and optimal design of electric machines and power apparatus. 